\title{
Measuring Multitasking Behavior with Activity-Based Metrics
}

\author{
RAQUEL BENBUNAN-FICH, Baruch College, City University of New York \\ RACHEL F. ADLER, The Graduate Center, City University of New York \\ TAMILLA MAVLANOVA, The Graduate Center, Baruch College, City University of New York
}

\begin{abstract}
Multitasking is the result of time allocation decisions made by individuals faced with multiple tasks. Multitasking research is important in order to improve the design of systems and applications. Since people typically use computers to perform multiple tasks at the same time, insights into this type of behavior can help develop better systems and ideal types of computer environments for modern multitasking users. In this paper, we define multitasking based on the principles of task independence and performance concurrency and develop a set of metrics for computer-based multitasking. The theoretical foundation of this metric development effort stems from an application of key principles of Activity Theory and a systematic analysis of computer usage from the perspective of the user, the task and the technology. The proposed metrics, which range from a lean dichotomous variable to a richer measure based on switches, were validated with data from a sample of users who self-reported their activities during a computer usage session. This set of metrics can be used to establish a conceptual and methodological foundation for future multitasking studies.
\end{abstract}

Categories and Subject Descriptors: H.5.2 [Information Interfaces and Presentation]: User InterfacesTheory and methods; H.5.3 [Information Interfaces and Presentation]: Group and Organization Interfaces-Evaluation/methodology; Theory and models

General Terms: Measurement

Additional Key Words and Phrases: Multitasking, metrics, activity theory, IT usage, task switching, user behavior

\section{ACM Reference Format:}

Benbunan-Fich, R., Adler, R. F., and Mavlanova, T. 2011. Measuring multitasking behavior with activitybased metrics. ACM Trans. Comput.-Hum. Interact. 18, 2, Article 7 (June 2011), 22 pages.

DOI $=10.1145 / 1970378.1970381$ http://doi.acm.org/10.1145/1970378.1970381

\section{INTRODUCTION}

Nowadays people drive while talking on the phone, check their email while eating lunch, or do their homework while chatting online with a friend. In this multitasking era, much has been said about this practice but relatively little comprehensive research has been conducted to date. This void might be due in part to the difficulty to conceptualize multitasking and subsequently measure it. Broad conceptualizations of multitasking are challenging because the existing body of literature appears to be divided into two separate streams with little cross-fertilization between them. On the one hand, studies on interruptions [Bailey and Iqbal 2008] address multitasking as the result of the decision to attend to an external notification. On the other hand, studies

Authors' addresses: R. Benbunan-Fich, Statistics and Computer Information Systems Department, Box 11220, Zicklin School of Business, Baruch College CUNY; email: Raquel.Benbunan-Fich@baruch.cuny.edu; R. Adler, Statistics and Computer Information Systems Department, Box 11-220, Zicklin School of Business, Baruch College CUNY; T. Mavlanova, Statistics and Computer Information Systems Department, Box 11220, Zicklin School of Business, Baruch College CUNY.

Permission to make digital or hard copies of part or all of this work for personal or classroom use is granted without fee provided that copies are not made or distributed for profit or commercial advantage and that copies show this notice on the first page or initial screen of a display along with the full citation. Copyrights for components of this work owned by others than ACM must be honored. Abstracting with credit is permitted. To copy otherwise, to republish, to post on servers, to redistribute to lists, or to use any component of this work in other works requires prior specific permission and/or a fee. Permissions may be requested from Publications Dept., ACM, Inc., 2 Penn Plaza, Suite 701, New York, NY 10121-0701 USA, fax +1 (212) 869-0481, or permissions@acm.org.

(C) 2011 ACM 1073-0516/2011/06-ART7 $\$ 10.00$

DOI 10.1145/1970378.1970381 http://doi.acm.org/10.1145/1970378.1970381 
on discretionary task interleaving [Payne et al. 2007] examine multitasking as the product of internal cognitive decisions to switch tasks. Yet multitasking is the result of both of these issues (external interruptions and internal choices) that lead people to change tasks. In this paper, we propose a comprehensive definition of multitasking and a set of metrics that enables us to examine prior empirical findings in a new light and to outline a new research agenda for multitasking.

Multitasking can be viewed as the result of time allocation decisions that individuals make when they are faced with multiple tasks. Thus a complete conceptualization of multitasking should look beyond the specific triggers that lead individuals to undertake multiple tasks and focus instead on actual time allocation decisions, as individuals shift their attention among ongoing tasks. A new definition along these lines lays the foundation for the development of specific measures to capture multitasking activity. Consensus on a broad definition allows the development of precise measures of multitasking, which are essential to research the antecedents and consequences of this behavior, particularly in terms of workers productivity and decision makers' performance [Speier et al. 2003].

Given modern tendencies to multitask with technology devices, computer-mediated activities provide an appropriate environment to examine multitasking from a conceptual and empirical perspective. Contemporary work and nonwork environments are characterized by people engaged in multiple ongoing activities spanning one or more technology devices. As some empirical studies report, there is a prevalence of multitasking during meetings [Benbunan-Fich and Truman 2009; Hembrooke and Gay 2003; Wasson 2004] or while at work [Czerwinski et al. 2004; González and Mark 2004; Mark et al. 2005]. Our study is focused only on a single IT platform (a personal computer) and the multitasking that occurs within this platform. While Human-Computer Interaction (HCI) research has begun to examine multitasking with multiple devices, such as using a smart phone while driving [Salvucci 2009], we believe that in order to understand why users switch among different devices, it is necessary to investigate first switches that occur within a single device. Furthermore, understanding the multitasking that occurs solely on a personal computer can be useful for interface designers. Given the tendency of modern users to have multiple unrelated windows open at any time, exploring how users multitask in this type of computer environment can help improve the design of systems and interfaces.

In this paper, we define multitasking in general and focus on computer-based multitasking to develop a set of metrics for this construct. The rest of the paper proceeds as follows. We start with a precise conceptualization of underlying principles of multitasking behavior, and then use Activity Theory as the foundation to address this topic from the perspective of the user, the task, and the technology. Based on this triad (usertask-technology), we proceed to the development of seven multitasking metrics. After presenting and discussing our proposed metrics, we report the results of an empirical study where these metrics were validated. We end by outlining potential areas for future research. Our aim is to develop an encompassing definition and set of metrics to organize existing findings and seed future research on this topic.

\section{WHAT IS MULTITASKING?}

Although multitasking is prevalent in everyday life, multitasking behavior has been difficult to conceptualize and measure. The very definition of multitasking as "multiple tasks performed at the same time," is open to several interpretations depending upon how tasks and time are defined [Rubinstein et al. 2001; Wild et al. 2004]. For example, tasks may include any combination of perceptual and motor activities, as in the typical example of using the cell phone while driving, and time may refer to short unit of time or a longer period. Similarly, tasks may be defined at a lower level of abstraction or at 
a higher level. Changes in the scale or granularity of these two dimensions will yield very different views of multitasking.

An accurate definition of multitasking starts with the articulation of two key dimensions: tasks and time. According to Wild et al. [2004: 18], "[w]hen understanding multitasking it is useful to scope the multiple 'what' and the overall duration of the "when." To scope tasks, it is useful to define them as self-contained units that incorporate all the components necessary for their performance. For example, working on a presentation might include shifting among an open browser for a Google search, Microsoft Word for viewing a paper, and Microsoft PowerPoint for writing the presentation itself. All of these components belong to the same task of preparing a presentation. For a more encompassing analysis, tasks are defined at a higher conceptual level, as opposed to a lower physical level. With this approach, tasks are complete and independent from each other. To scope time, it is preferable to use the notion of a session with a beginning and an end, instead of a standard unit of time, such as an hour. The scoping of a time frame in terms of a session allows for a more comprehensive examination of tasks and their performance, as some tasks may not be completed in predefined standard units of time.

A complete conceptualization of multitasking also requires an understanding of how tasks are performed. Depending on differences in time allocation decisions, there are several approaches for the temporal organization of multiple independent tasks in a segment of time. At one extreme, multiple tasks can be executed in sequence by starting each task after the completion of another. In this sequential mode, only one task is attended to at a time from beginning to end. Even though multiple tasks might be performed in a time period, there is no concurrency. At the other extreme, multiple tasks can be executed in parallel, if all of them are ongoing at the same time. In this case, there is maximum concurrency among tasks. In practice, however, absolute parallel performance might not be possible as humans are unable to simultaneously divide their attention among ongoing tasks [Bluedorn et al. 1992]. The middle ground between these two extremes is interleaving tasks by diverting attention or switching from one task to another and eventually resuming previously abandoned tasks [Payne et al. 2007]. This interspersing strategy is common in computer-based multitasking, where tasks under way are voluntarily or involuntarily suspended to perform other tasks. This type of multitasking involves a partial degree of concurrency among tasks performed in a segment of time.

We propose that the principles of task independence and performance concurrency are central to achieve a precise conceptualization of multitasking. Bearing these two principles in mind and consistent with our focus on a single IT platform, we adopt the following definition: A user engages in computer-based multitasking behavior when s/he performs more than one unrelated computer-based task concurrently. Computerbased multitasking is thus a function of time allocation decisions. Such decisions determine how multiple independent tasks are combined in a segment of time and the degree of concurrency with which these tasks are performed in that period of time. According to this definition, a comprehensive view of multitasking encompasses the components of the triad identified by Burton-Jones and Straub [2006] to measure IT usage: usertask-technology. Our metrics development effort is grounded on the analysis of these three elements from the perspective of Activity Theory.

\section{THEORETICAL BACKGROUND}

Activity Theory is a psychological framework or meta-theory that integrates motivation, cognition and behavior in the context of human practices [Bedny and Karwowski 2007; Kaptelinin and Nardi 2006]. Based on a set of principles that describe the hierarchical nature of human activities and the role of tools in human thought and 
behavior, Activity Theory seeks to understand the unity of consciousness and activity, as evidenced in the interaction with other people or artifacts. Due to its focus, it has been applied to the study of Information Technologies, Systems Design, and HumanComputer Interaction. This theory emphasizes human intentionality while recognizing that an activity is a combination of prespecified and situated components [Kaptelinin and Nardi 2006]. This distinction is particularly important when people act with technologies [Kaptelinin and Nardi 2006], because the technology facilitates and constrains the attainment of the user's goals, and the components of an activity can dynamically change as conditions change. ${ }^{1}$

Two broad trends can be identified in the application of Activity Theory to Information Technology design and Human-Computer Interaction [Bedny and Harris 2008]. The Scandinavian perspective [Bødker 1989; Kuutti 1991; Kaptelinin 1996; Nardi 1996], and the systemic-structural perspective developed by Bedny and colleagues [Bedny et al. 2000; Bedny and Harris 2005; Bedny and Karwowski 2007]. While researchers in the Scandinavian tradition of Activity Theory emphasize the principle of object-orientation whereby an activity is a unit of subject-object interaction [Kaptelinin and Nardi 2006], the systemic-structural approach is more focused on conscious goals as drivers of activity. In the systemic-structural perspective, an activity is defined as a "goal directed system in which cognition, behavior and motivation are integrated and organized by goals and the mechanisms of self-regulation" [Bedny et al. 2000, p. 168]. We use goal-orientation as one of the key principles of Activity Theory.

In Activity Theory, an activity is defined at a high level and consists of actions and operations. Within this hierarchical conceptualization, each activity consists of several actions, which in turn involve several operations. The top layer of this hierarchical structure contains the activity itself, which is oriented towards a motive or object that the subject needs to attain [Kaptelinin and Nardi 2006]. The second layer contains actions, or functionally subordinated processes, directed at specific conscious goals. The third layer consists of operations, which result from the decomposition of actions. These lower level units are routine processes that do not require a conscious effort for their execution but allow adjustment to ongoing circumstances. Operations are automatic responses to perceived conditions that allow humans to adjust their actions to the current situation [Nardi 1996; Kaptelinin 1996]. The combination of goal orientation with dynamic adaptation in human activities accounts for predictable and routine events, as well as emergent and contingent actions.

Another key premise in Activity Theory is the notion of mediation by artifacts or tools that enable humans to carry out specific activities [Kuutti 1991]. A tool mediates an activity that connects a person not only with the world of objects, but also with other people. Tool mediation allows humans to extend their capabilities and perform new functions, or perform existing functions more efficiently [Kaptelinin 1996]. For instance, people use computers to extend their information processing or their communication capabilities. In so doing, they seek to achieve objectives that are meaningful beyond the actual use of the computer [Kaptelinin 1996].

With an explicit articulation of humans, activities and tools, this framework is suitable to analyze each of the components of the triad (user-task-technology) and understand multitasking. The basic principles of Activity Theory, along with specific

\footnotetext{
${ }^{1}$ The conceptual apparatus of Activity Theory is more extensive than the discussion presented herein. We focus on the main tenets of this theory, the two main schools of thought within this theory and how they contribute to the study of multitasking. A more detailed explanation of this theory is available in Bedny et al. [2000], Bedny and Karwowski [2007], Kaptelinin and Nardi [2006], Kuutti [1991], and Nardi [1996]. A discussion of how to use Activity Theory in HCI research is presented in Kuutti [1996] and in Bedny and Karwowski [2003].
} 
analytical elements developed in the systemic-structural perspective provide the theoretical underpinnings for the study of multitasking. In the systemic-structural approach, human work activity during task performance is the primary object of study. To understand human work activity, this approach includes morphological and functional analysis techniques. In morphological analysis, the structure of an activity is conceptualized as a logical and spatiotemporal organization of actions and operations performed to achieve the goal of a task. In functional analysis, the major units of analysis are function blocks and an activity is regarded as a self-regulative or adaptive system that actively interacts with the situation [Bedny and Karwowski 2007].

Building on the principles of goal orientation, hierarchical conceptualization, technology mediation, and additional elements provided by the systemic-structural approach, we analyze the interplay of user, task and technology. Together these principles lay the theoretical foundation for the development of multitasking metrics from the perspective of Activity Theory.

\subsection{Task Perspective}

A task is an assigned or voluntary undertaking that requires time and other resources to produce an outcome. Typical task conceptualizations are based on an input-processoutput model and thus include the required steps and information cues necessary (input) to create a product or achieve a goal, which is considered an output. The process whereby task inputs are transformed into outputs can be articulated in terms of procedural components identified as subtasks and steps [McGrath 1991; Wood 1986].

In the systemic-structural approach of Activity Theory, a task is a fundamental component [Bedny and Harris 2005]. While activities respond to needs and motives that are often subconscious, tasks have specific conscious goals. Any task may be represented as a logically organized system of actions around an overall goal. In turn, each action has separate but intermediate goals that must be reached to attain the overall goal of the task [Bedny et al. 2000]. ${ }^{2}$ By inserting tasks in a middle layer between activity and actions, tasks provide a vehicle to organize actions (or subtasks) around a high level goal. This definition is consistent with the two parameters we used to scope tasks. First, it includes within each task all the components necessary for their performance so that tasks are complete and self-contained. Second, it operates at a high level of abstraction determined by a goal, which is consistent with our scoping of tasks at a conceptual level.

Our application of Activity Theory to the context of multitasking involves broadening the focus from a single task to multiple independent tasks performed concurrently. From the perspective of the task, multitasking manifests itself when there is a task switching decision (i.e., a task currently underway is temporarily abandoned to undertake or continue another task). In the systemic-structural perspective, task switching results from the temporal structuring of activity elements and their sequential or simultaneous performance [Bedny and Karwowski 2007]. Other Activity Theory researchers have conceptualized switching in terms of focus shifts [Bødker 1996]. Changes in focus can be deliberate or caused by breakdowns. According to findings in psychology [Payne et al. 2007], discretionary focus shifting (or voluntary task interleaving) is motivated by two task-related factors: (1) diminished rate of return: propensity to temporarily abandon a task that is no longer rewarding; or (2) subgoal completion: tendency to switch to an unrelated task when a sub-task is completed. Although these two factors can be considered task-related, they signal a change in the user's original goal-orientation. Hence, the user's perspective offers a complementary view of multitasking.

${ }^{2}$ We are very grateful to the anonymous reviewers of this journal for directing us to the work of Bedny and colleagues and for helping us articulate the distinction between tasks and activities in Activity Theory. 


\subsection{User Perspective}

Activity Theory considers humans as purposeful in terms of motives and goals. Activities are oriented to general needs, while tasks are associated with specific goals. Individuals usually participate in several activities at the same time [Kuutti 1996]. Hence an application of this theory supports the view of individuals undertaking different tasks to fulfill multiple goals (i.e., multiple goal orientation) with freedom to decide how many tasks will be performed and how they will be combined. Each individual's interpretation and performance of a task is to some extent idiosyncratic [Bedny et al. 2000], as different people may understand the same task differently or may develop different strategies to complete a task [Hackman 1969; McGrath 1991]. Similarly, each individual may approach the decision to multitask in various ways, including not only which tasks to perform but also how to integrate their performance in a particular time interval. Users typically break down high-level tasks into smaller components (subtasks), which are then executed and potentially combined with components of other ongoing tasks, sequentially, simultaneously, or by alternating them. Temporal structuring of activities allows for multiple combinations in their integration into a dynamic whole [Bedny et al. 2005].

Concurrent task performance and voluntary task switching decisions may result from factors internal to the user, such as individual characteristics or personality traits that motivate people to undertake unrelated activities in the same time interval and switch between them. One way to capture people's propensity to multitask is to measure their time use preferences and behavior. Based on Hall's [1983] concepts of monochronic and polychronic time use, an individual with monochronic time preferences will tend to focus on one thing at a time, while an individual with polychronic tendencies will tend to simultaneously undertake two or more activities [Lee 1999]. Instead of a dichotomy, Bluedorn et al. [1992] argue that there are degrees of polychronicity, ranging from people who tend to be very monochronic to those who are very polychronic. Given personal time use preferences, polychronic individuals will be more likely to engage in multiple tasks, while monochronic individuals will be inclined to perform one task at a time.

From the user's perspective, a necessary condition for multitasking is multiple goal orientation. The need to fulfill multiple goals may explain the user's tendency to undertake multiple tasks in a particular time interval. However, due to personal characteristics and time orientation preferences, each user may adopt different temporal strategies for the execution of multiple tasks and their subcomponents ranging from pure sequential task performance to interspersing subtasks. Therefore, a sufficient condition for multitasking is the temporal integration of components from various tasks so that multiple task performance takes place within a particular time interval. Temporal integration occurs when the user decides to switch from one subtask to another subtask that belongs to a different task. The ability to change from one task that is in progress to another one is influenced by the technology tools that mediate task performance.

\subsection{Technology Perspective}

Activity Theory supports the view of technology as a mediating tool that allows individuals to carry out tasks. In a single task context, user performance of technologymediated tasks is visible through the interaction between the user and the technology. In this interaction process, a user not only initiates actions to perform task-related steps but also responds to technology prompts, such as a notification that a relevant document has finished downloading. Electronic notifications emerging from the technology itself encourage users to respond to these contextual signals for the purposes of self-regulation. 
In modern computer-based environments, the computer is the means of work and cannot be classified as a single tool. Instead, it should be conceptualized as a way of presenting a variety of tools that mediate task performance [Bedny and Harris 2005]. User-technology interaction consists of employing programs or applications rendered in windows. Applications are provided by the technology to allow task performance. Typically, a high-level task is broken down into functional components, which are in turn associated with specific technology tools or applications for its performance. Functional association is the process whereby task components are related to specific technology tools. For instance, a task that requires information gathering and summarization consists of two functional subtasks performed by using two independent applications and two or more separate windows, namely: conducting online research (with a Web browser) and writing up the information (with a word processor).

Technology mediation plays a dual role in the performance of multiple concurrent tasks. On the one hand, contemporary computing platforms have been explicitly designed to support multitasking. From the ability to toggle between windows, which allows users to keep different programs or files open and alternate among them, to the availability of browser tabs that let users conduct multiple activities within a web browser window, modern personal computing facilitates multitasking. On the other hand, the very same technology that enables multitasking tends to intensify it, as users are interrupted by programs or electronic notifications ${ }^{3}$ that may lead (or force) them to switch tasks unexpectedly. Notifications are a specific technology feature designed to keep users contextually aware of changes in their computer-based environment [McCrickard et al. 2003]. Thus, personal computing technology acts as an enabler of multiple task performance and as a potential multitasking inducer as well.

As an enabler, the technology allows users to work concurrently on multiple outstanding tasks as they await responses from others concerning these tasks [Whittaker 2005]. The need to constantly wait for responses, or perhaps the need to be constantly available for others, explains why people tend to keep email running in the background. Renaud et al. [2006] found that $84 \%$ of the people kept email running in the background at work, while $55 \%$ of them had email running in the background at home. Although about half of the users in their sample used alerts to get notified of new incoming email messages, usage tracking showed that most people voluntarily switched to check email about every five minutes [Renaud et al. 2006]. Consistent with these findings, González and Mark [2004] report that office workers tend to switch between different working spheres due to either self-interruptions or external interruptions. In related research, Reinsch et al. [2008] discuss the practice of multicommunicating or carrying overlapping technology-mediated conversations in the workplace.

As an inducer, the technology prompts users to switch tasks when they receive notifications from computer-mediated communication systems, such as email and instant messaging software, or alerts from their computers (e.g., schedule reminders). The interruptions literature provides a rich context to understand why people decide to switch tasks and the implications of this decision. For example, while Gillie and Broadbent [1989] conclude that interruptions have a deleterious effect on performance, Cutrell et al. [2000] found that interruptions that are pertinent to current tasks are less disturbing than those that are irrelevant. Speier et al. [1999] found that interruptions facilitate performance on simple tasks, while inhibiting performance on more complex

\footnotetext{
${ }^{3}$ Although interruptions may come from other devices (such as smart phones, cell phones, pagers, etc.), or from other people, our interest here is on interruptions that occur within the same personal computing platform (laptop or desktop) where the user is carrying out his/her work. In a recent study, Ophir et al. [2009] coined the term "media multitasking" to distinguish consumption of content from multiple devices from a situation where multiple streams of content come from a single device.
} 
tasks. For complex tasks, the frequency of interruptions and their lack of similarity with the task at hand exacerbated the negative performance effects. Speier et al. [2003] confirm these findings but suggest that interruptions may have positive effects by allowing people to break from a difficult task and approach it later with a different mindset. In addition to task complexity, the timing of the interruption and the way it is handled is germane [McFarlane 2002]. With respect to timing, the effects on performance are less severe when the interruption occurs at subtask boundaries [Bailey and Iqbal 2008; Hodgetts and Jones 2006; McFarlane and Latorella 2002; McFarlane 2002; Payne et al. 2007]. It is worth noting that not all interruptions are mandatory. In some cases, people exercise their discretion to handle interruptions at a later more convenient time [McFarlane 2002].

This dual view of technology that not only supports but also generates multitasking is consistent with the dynamic adaptation principle of Activity Theory. According to this principle, goal-oriented individuals are able to adapt to dynamic changes in their environment and respond accordingly. Users can dynamically adjust or change tasks as a result of technology notifications. As users remain contextually sensitive, technology signals might affect the order in which they carry out subtasks (within the same task) or might prompt them to switch to a completely different task. Technologymediated activities tend to incorporate prespecified and emergent task components. In particular, users pay attention to and decide whether to respond to electronic alerts and interruptions. Therefore, multitasking is not only an individual choice but also the result of the dynamic adaptation of users to the technology environment in which they carry out their tasks.

\section{ACTIVITY-BASED MULTITASKING METRICS}

Using the principles of Activity Theory and our application of this theory for multitasking, we organize existing metrics for computer-based multitasking behavior and propose new ones in a systematic way, using the triad (user, task, and technology) identified by Burton-Jones and Straub [2006] to articulate metrics for technology usage. Accordingly, these metrics are developed and discussed with respect to the user, task or computer technology perspective, and a progression from leanest to richest depending on how many elements are considered in each measure. Heretofore, we identify each measure of multitasking (MT) with subscripts to indicate its relation to the user (U), task (T), or computer technology (C).

The proposed multitasking metrics are objective and computer-centric. Objective because they are based on actual usage patterns, rather than the user's perceptions of their own multitasking habits. Computer-centric because they consider a single device (personal computer) and include only computer-based tasks. Thus all other manual or perceptual tasks performed by users (such as watching TV or talking on the phone while using the computer) are excluded, and all other technology devices, such as smart phones and handheld gadgets that might be used concurrently with a laptop or desktop computer, are omitted.

Activity theory emphasizes human intentionality and thus conceptualizes users as goal-oriented in the performance of tasks. As each task is associated with a highlevel goal, from the perspective of the user, multitasking can be measured with a dichotomous variable. This measure $\left(\mathbf{M} \mathbf{T}_{\mathrm{U}}\right.$ ) would indicate whether or not an individual is performing multiple goal-independent tasks concurrently in a specific period of time (or in a computer session). This is a very lean measure of multitasking. It can be obtained directly from the user or indirectly by observing his/her performance and it can be used as a control variable in other studies focused on technology usage.

$M \boldsymbol{T}_{U}=\boldsymbol{O}$ or 1, where 0 is mono-tasking and 1 is multitasking. 
Our application of Activity Theory to the context of multitasking defines tasks as an instrument to organize actions around a high-level goal. In this conceptualization, a more informative but still lean measure of multitasking focused on tasks $\left(\mathbf{M T} \mathbf{T}_{\mathrm{T}}\right)$ would indicate the extent to which multitasking is occurring by measuring the number of unrelated tasks per session time. Prior studies have used this type of taskrelated metric. For example, Wild et al. [1994] propose to measure multitasking with the number of parallel and interleaved tasks that are carried out over a period of time.

$\boldsymbol{M} \boldsymbol{T}_{T}=\Sigma \boldsymbol{t}_{i}$, where $t_{i}$ is an independent task carried out during the session.

According to the tool-mediation principle of Activity Theory, the technology enables task performance. In a computer-based environment, this functional view applies to the different software programs used. Therefore, from the perspective of the computer technology, multitasking $\left(\mathbf{M} \mathbf{T}_{\mathrm{C}}\right)$ can be measured by the number of applications or software programs used during a computer session [e.g., Crook and Barrowcliff 2003]. The assumption for this metric is that different applications are used for unrelated tasks, which may not hold in all situations. For example, a user can be taking information from the Internet to write an essay. In this case, the browser and the word processor are two different applications used for the same high-level task (essay writing). Because of its exclusive focus on applications, this is an appropriate measure from the technology perspective.

$\boldsymbol{M} \boldsymbol{T}_{C}=\Sigma \boldsymbol{a}_{i}$,

where $a_{i}$ is an application or software program.

In order to develop richer measures of multitasking, we combine at least two elements of the triad and develop new principles based on Activity Theory. Accordingly, we identify three pairs: User-Technology, Task-Technology and User-Task.

For the User-Technology pair, a metric indicating how many windows ${ }^{4}$ are used (or active) during a computer session (MT $\mathbf{T}_{\mathrm{UC}}$ ) would give an indication of the interaction between the user and the computer. A count of active windows gives a more precise indication of the way the user interacts with the technology than just the number of applications as multiple instances of the same application can be used to perform distinct tasks. However, it is necessary to make a distinction between open windows and active windows. Users may have open windows that they are not attending to, or neglect to close them after they finish and leave a trail of open windows. In contrast, active windows are those that an individual is using by switching to and from them during his computer session. Therefore, the notion of active windows captures both the user's perspective as well as the technology perspective.

$\boldsymbol{M} \boldsymbol{T}_{U C}=\Sigma \boldsymbol{w}_{i}$, where $w_{i}$ is a window from an application or a browser tab.

Activity Theory is based on the hierarchical conceptualization of activities and the structuring of tasks in terms of its components (subtasks and steps). When the performance of tasks is mediated by technology tools, this decomposition process is not only hierarchical but also functional. Functional decomposition consists of selecting specific technology tools (or applications) to perform the subordinated components of each task. For instance, a person could be using a Web-based application to monitor the status of

\footnotetext{
${ }^{4}$ In this article, we use the term "window" broadly to indicate the active portion of the screen. Therefore, the term encompasses not only application-related windows but also browser tabs, which are typically nested within a single window but activated at different times.
} 
her bank account in online banking along with a local spreadsheet program to balance her checkbook. Therefore, a multitasking measure applicable to the Task-Technology pair $\left(\mathbf{M T}_{\mathrm{TC}}\right)$ would give an indication of the number of different software programs or applications required for each task. One way to capture this functional association between tasks and applications across all tasks is by dividing the number of applications by the total number of tasks.

$\boldsymbol{M} \boldsymbol{T}_{T C}=\left(\Sigma \boldsymbol{a}_{i}\right) /\left(\Sigma \boldsymbol{t}_{j}\right)$,

where $\Sigma a_{i}$ is the number of applications and $\Sigma t_{j}$ is the total number of tasks.

From the perspective of the User-Task pair, the temporal integration of task components related to multiple independent tasks results in a richer multitasking indicator $\left(\mathbf{M T}_{\mathrm{UT}}\right)$. This metric is based on the percentage of overlapping tasks. A task (t) is considered an overlapping task $(\theta \mathrm{t})$ when its start time occurs before the end time of a previously started task. The percentage of overlapping tasks depends on the general strategy employed by a user to execute multiple tasks in a period of time. For example, strictly sequential users would have no overlapping tasks while those who work in parallel will have a high percentage of tasks overlapping. As such, this metric reflects different temporal integration strategies when multiple tasks are performed.

$\boldsymbol{M} \boldsymbol{T}_{U T}=\left(\Sigma \theta \boldsymbol{t}_{i}\right) /\left(\Sigma \boldsymbol{t}_{j}\right)$ where $\Sigma \theta t_{i}$ is the total number of overlapping tasks and $\Sigma t_{j}$ is the total number of tasks.

While the percentage of overlapping tasks is a promising metric, it can be improved by incorporating the number of times that the user actually shifts attention between overlapping tasks. Prior studies have defined and measured multitasking as rapid task switching [Bell et al. 2005] or simply with a count of task switches in a specific time period [Czerwinski et al. 2004].

When tasks are performed in a computer-based environment, a decision to change tasks involves changing active windows (or active tabs within a window). However, not every change in the active window is indicative of a change in tasks. Therefore, it is necessary to distinguish between two types of switches: within-task switches and between-task switches. Wild et al. [2004] make this distinction based on whether the primary task changes when there is a switch. In this study, we define within-task switches (Sw) as changes of windows while performing the same task and between-task switches $(\mathrm{Sb})$ as window changes that indicate a change in the tasks being performed. While in both cases there is a change in the active window, it is only in the latter that there is a change in task.

The most comprehensive and richest measure we propose $\left(\mathbf{M T}_{\text {UTC }}\right)$ includes UserTask-Technology considerations. This metric consists of calculating the proportion of between-task switches $(\mathrm{Sb})$ with respect to all switches $(\mathrm{Sb}+\mathrm{Sw})$. This ratio gives an indication of the extent to which users perform different tasks concurrently, as it considers how the user changes focus from one task to another by changing active windows. To the best of our knowledge, this metric is new and has not been used before in the literature. Furthermore, this measure offers a more precise account of multitasking behavior by incorporating the two principles of performance concurrency and task independence we used to define multitasking.

$\boldsymbol{M} \boldsymbol{T}_{U T C}=\left(\Sigma \boldsymbol{S}_{b}\right) /\left[\left(\Sigma \mathbf{S}_{b}\right)+\left(\Sigma \boldsymbol{S}_{w}\right)\right]$,

where $\Sigma S_{b}$ is the total number of between-task switches and $\left[\left(\Sigma S_{b}\right)+\left(\Sigma S_{w}\right)\right]$ is the total number of switches.

Figure 1 summarizes our proposed multitasking measures in terms of their relation to each element of the triad (user, task and computer technology). 
$\operatorname{USER}(\mathbf{U})$

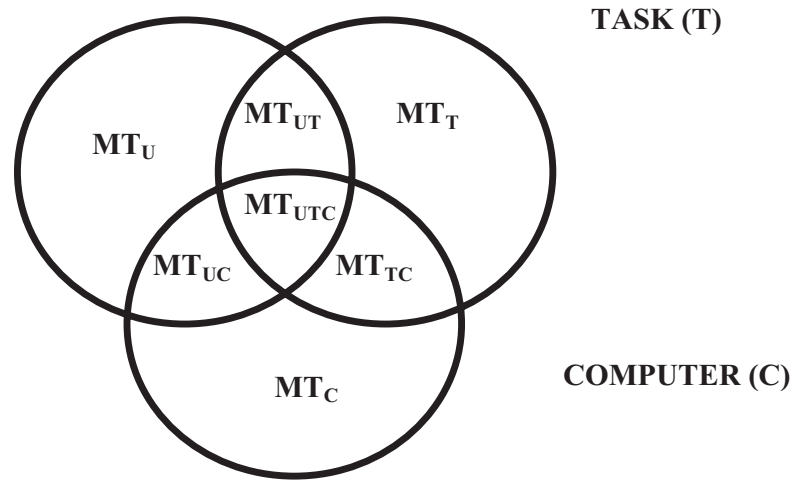

Fig. 1. Activity-based multitasking metrics.

\section{RESEARCH METHODS}

In order to examine the validity of these multitasking indicators and discuss their possibilities and limitations, we collected a sample of user self-reports of home computer usage. A home setting allows for an investigation of discretionary computer usage with more freedom for choosing tasks and switching among them than an office environment. Furthermore, a home setting lacks constraints related to social norms of appropriate computer use, or more explicit access restriction mechanisms, which might be prevalent in the workplace. User-produced reports of computer usage (or diaries) have high ecological validity but their production may disrupt typical computer-based activities. On the upside, diary studies or user-generated logs are produced by the respondents themselves as the events take place and do not suffer from problems of recall. The downside is that the process of recording activities may be tedious for participants and affect their normal flow of work [Czerwinski et al. 2004].

Participants were asked to self-report in detail their computer-based activities for one session of their computer usage of about one to two hours. To minimize interference between log record keeping and normal computer-based activities, we asked subjects to annotate manually all of their switches on a standard log table provided on a paper form. The form consisted of a table with six columns: time, action (open/close/return), application, window (or tab) with file name or Website name (if applicable), purpose on window, and reason for going to another window. Filling out the log in their computers was not an option because this additional activity may inflate some of the multitasking metrics. In addition, to mitigate potential problems associated with tedium, we asked users to log only one session of computer usage of about one or two hours. We decided to collect self-reports despite the limitations of the method because self-reported logs allow users to describe their tasks and goals in their own words and to provide the rationale for shifting focus among different activities.

This exercise was implemented as an assignment in an undergraduate introductory course in Information Systems taught at a large urban college located in the Northeast of the United States (Appendix A shows the instructions given to participants). Upon submission to their instructor, the students were asked by a researcher not connected to the course to donate their assignments for research. Those who agreed to participate signed a consent form and made their assignments available to the researcher. No incentives were given to encourage students to participate in this project.

\section{DATA ANALYSIS}

We collected a total of two hundred logs from participants who agreed to make their assignment available for research. Several logs had to be discarded due to session 
Table I. Descriptive Statistics of Variables

\begin{tabular}{|c|c|c|c|c|}
\hline Sample Size & $\mathrm{n}=14$ & & & \\
\hline Dichotomous Multitasking $\left(\mathrm{MT}_{\mathrm{U}}\right)$ & No $=?$ & Yes $=76$ & & \\
\hline & Mean & St. Dev & Min & Max \\
\hline Session Time (mins) & 69.88 & 15.54 & 50 & 122 \\
\hline Number of Tasks $\left(\mathrm{MT}_{\mathrm{T}}\right)$ & 4.60 & 1.87 & 1 & 9 \\
\hline Number of Applications $\left(\mathrm{MT}_{\mathrm{C}}\right)$ & 2.87 & 1.49 & 1 & 8 \\
\hline Number of Applications by Task $\left(\mathrm{MT}_{\mathrm{TC}}\right)$ & 0.77 & 0.61 & 0.14 & 3 \\
\hline Number of Windows $\left(\mathrm{MT}_{\mathrm{UC}}\right)$ & 5.78 & 2.96 & 2 & 16 \\
\hline Number of Overlapping Tasks $(\theta t)$ & 2.80 & 2.01 & 1 & 8 \\
\hline Total Number of Switches & 13.66 & 7.33 & 3 & 36 \\
\hline Number of Between-Task Switches & 4.64 & 4.74 & 0 & 23 \\
\hline Percentage of Overlapping Tasks $\left(\mathrm{MT}_{\mathrm{UT}}\right)$ & 55.1 & 28.4 & 0 & 88.9 \\
\hline Percentage of Between-Task Switches (MT $\left.\mathrm{MTC}_{\mathrm{UT}}\right)$ & 30.1 & 20.6 & 0 & 81.8 \\
\hline
\end{tabular}

length issues, compliance with reporting instructions or deviation from setting (for example, logging a session while at work). After discarding these reports, the final sample consists of 146 logs of continual computer use. We included logs that ranged from one to two hours plus or minus ten minutes, which resulted in session durations of 50 to 130 minutes (mean of $69.88 \mathrm{~min}$. and a standard deviation of $15.84 \mathrm{~min}$.). Appendix B shows an example of a log.

Two independent raters initially coded the logs to determine whether the user was multitasking according to the principles of performance concurrency and task independence. Inter-coder reliability results calculated with the total percentage of agreement in log classification was $80 \%$ (117 logs with consistent coding divided by total number of logs 146). Most of the discrepancies occurred because several logs did not contain enough detail to determine whether some activities were related or unrelated to each other (i.e., task independence). Additionally, it was not clear in some logs whether some tasks actually overlapped or were concluded without closing the appropriate windows (i.e., performance concurrency). Coder discrepancies were solved by discussion with a third coder. After the reconciliation process, it was agreed that 76 users (52\%) were multitasking $\left(\mathbf{M T} \mathbf{T}_{U}=1\right)$ and the remaining 70 users $(48 \%)$ were not $\left(\mathbf{M T}_{U}=0\right)$. This indicator produced by human coders is used to validate the remaining multitasking variables, which were determined automatically by a custom-developed software program.

We developed a Perl program to automatically parse and code the logs. By searching for action, task and application keywords in the users' log tables, the program calculated for each user in the sample the following variables: number of independent tasks $\left(\mathbf{M T} \mathbf{T}_{\mathrm{T}}\right)$, number of applications $\left(\mathbf{M T} \mathbf{T}_{\mathrm{C}}\right)$, number of windows $\left(\mathbf{M T} \mathbf{T}_{\mathrm{UC}}\right)$, number of overlapping tasks $(\theta \mathrm{t})$ and number of between- and within-task switches (Sb and $\mathrm{Sw}$ respectively). Automatically generated codes were compared with those produced by human coders for a sample of logs and no inconsistencies were detected.

Three additional multitasking metrics were calculated as a result of variables computed by the log-analysis program. $\mathbf{M} \mathbf{T}_{\mathrm{TC}}$ was obtained from dividing number of applications by tasks. $\mathbf{M} \mathbf{T}_{\mathrm{UT}}$ was calculated as the ratio of overlapping tasks to total tasks. $\mathbf{M T}_{\mathrm{UTC}}$ was computed as the percentage of between-task switches with respect to total switches (i.e., from dividing between-task switches $(\mathrm{Sb})$ by total number of switches $(\mathrm{Sb}+\mathrm{Sw}))$.

\section{RESULTS}

Table I presents the basic statistics for each variable. More detail on each variable is provided in what follows.

$\mathbf{M T}_{\mathrm{T}}$ was computed by the log analysis program by counting the number of independent tasks that the user performed during the session. Consistent with the principle 
of hierarchical conceptualization, tasks were defined at a higher level (e.g., checking email, doing homework, playing music, etc.). We found that the subjects reported their tasks at a similar level. (Appendix $\mathrm{C}$ shows a list of computer-based tasks most frequently found in the logs). All but seven subjects performed more than one unrelated task during their sessions. These seven participants' monotask consisted of doing homework, either by using the courseware system, a publisher's website and an excel spreadsheet to complete a quantitative assignment, or by using the courseware system, a word processor and Internet searches to write an essay. Although a single task was performed, multiple applications were used in each case. It should be noted due to its exclusive focus on number of tasks, this metric does not distinguish between a user who undertakes five tasks sequentially and another user who performs five interleaved tasks.

$\mathbf{M T}_{\mathrm{C}}$ was determined by counting the different software programs, such as Word Processor, Web Browser, Spreadsheet, Music Player and others, that were used throughout the session. Twenty four participants were using only one application during their session (their Web Browser) but all of these participants were performing more than one unrelated task ranging from searching to checking email or watching videos. On average, the number of applications used in a session is almost 3 . We found that a typical academic task (or homework) usually involves the use of more than one application (e.g., Web Browser and Word Processor for essays or Spreadsheet Software and Word Processor for analysis and report of numeric data).

$\mathbf{M T}_{\mathrm{UC}}$ was computed by counting the number of windows from software programs and browser tabs, used during the session. This metric indicates the extent to which an individual distributes his work in different windows and thus measures user-computer interaction. The number of windows ranges from 2 to 16, with an average of almost 6 . In this calculation, browser tabs are considered different windows even though they are technically nested within a single window. Similarly, each window is counted separately regardless of their task association.

$\mathbf{M T}_{\mathrm{TC}}$ was obtained by dividing the number of applications by the number of tasks. The results of this metric (mean of 0.77 and standard deviation of 0.61 ) indicate that on average, more than one task is performed with each application. The most illustrative example of this situation is the use of the Web browser for different purposes such as checking email, watching videos, reading Web-based news, searching for information, etc. In terms of the functional decomposition principle, one task may span multiple applications, and one application (such as the browser) may be associated with components of several tasks.

$\mathbf{M T}_{\mathrm{UT}}$ is measured with the percentage of overlapping tasks with respect to the total number of tasks. ${ }^{5}$ The percentage of overlapping tasks ranges from 0 to $88 \%$ with a mean of $55 \%$ and a standard deviation of $28 \%$. This metric gives an indication of the temporal organization of the tasks adopted by each user. At low overlapping, we find users who work in a strictly sequential way with very little or no overlap among independent tasks. In our sample, thirteen users showed a pure sequential behavior such as the one depicted in Figure 2(a). In this graph, produced by the log-analysis program, the lines indicate the amount of time (in minutes) that each task is active based on its start time and end time.

At high overlapping, we find users who carried out most of their tasks with high degree of concurrency. This pattern is shown in Figure 2(b). This user kept email and a calendar application active throughout the session, and in addition s/he was chatting and playing music, or chatting and watching videos.

\footnotetext{
${ }^{5}$ Consistent with our definition of overlapping tasks, the maximum number of overlapping tasks is: total tasks -1 , as the first task never overlaps a previously started task. Therefore, the percentage of overlapping tasks can never reach $100 \%$.
} 
Ho Overlap / Sequential (Log 24J13)

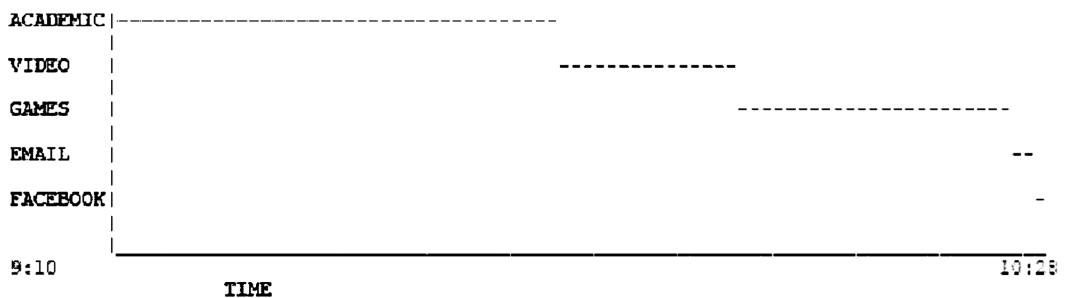

(a)

Iligh Overlap (Log 24001)

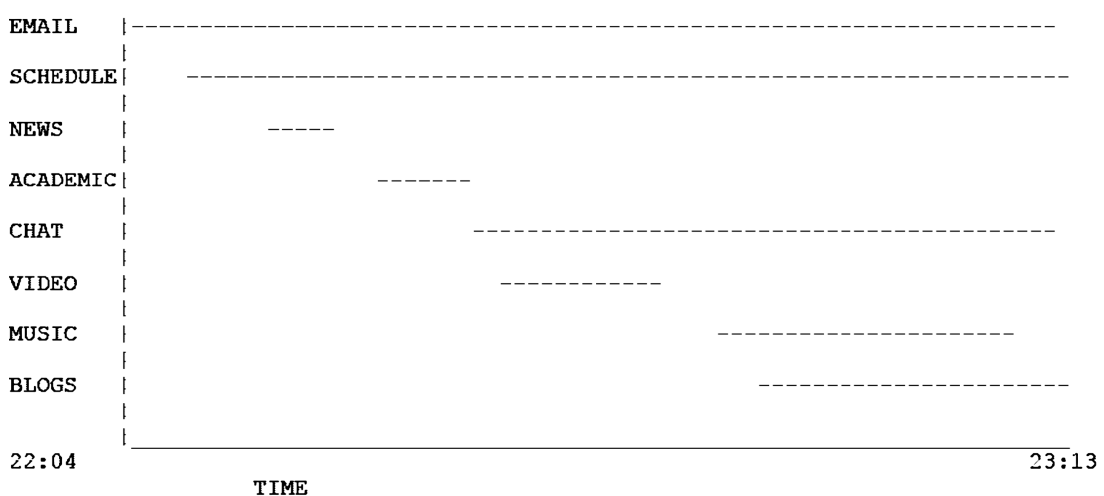

(b)

Medium (508) Overlap (Log 24026)

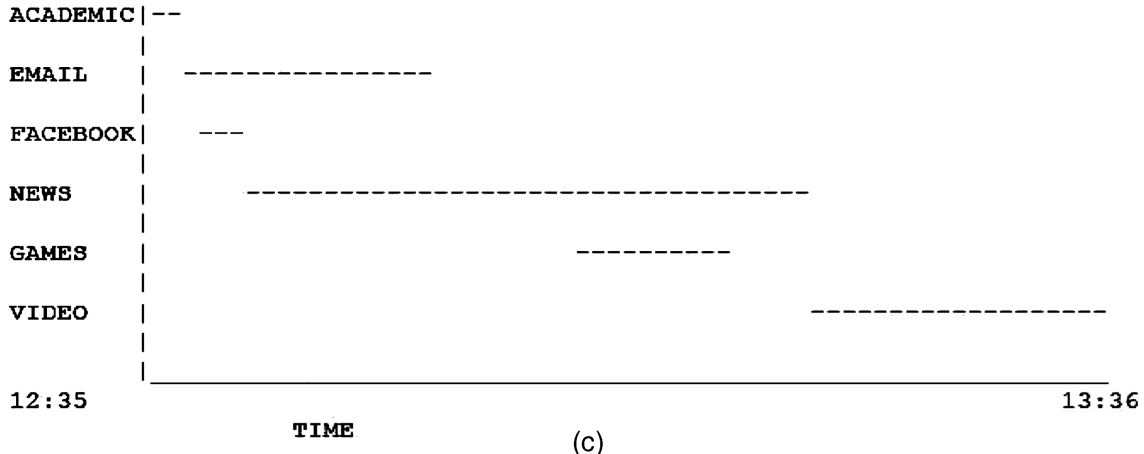

Fig. 2.

Figure 2(c) shows an example of a user with a medium level of task overlapping. This case is also illustrative of a mixed multitasking pattern, where some tasks are carried out sequentially (e.g., academic and email), while other tasks partially overlap (e.g. news and games).

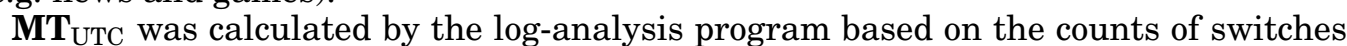
within a task and between tasks in each log. The percentage of between-task switches 
Table II. Correlation Table

\begin{tabular}{|c|c|c|c|c|c|c|c|}
\hline & $\begin{array}{l}\text { Session } \\
\text { Time }\end{array}$ & $\mathbf{M} \mathbf{T}_{\mathrm{T}}$ & $\mathbf{M T}_{\mathrm{C}}$ & $\mathbf{M T}_{\mathrm{TC}}$ & $\mathbf{M T}_{\mathrm{UC}}$ & $\mathbf{M T}_{\mathrm{UT}}$ & $\mathbf{M T}_{\mathrm{UTC}}$ \\
\hline Session Time (mins) & 1.00 & -0.02 & 0.05 & 0.04 & $\mid-0.03$ & 0.02 & 0.05 \\
\hline $\begin{array}{l}\text { Number of Tasks } \\
\left(\mathrm{MT}_{\mathrm{T}}\right)\end{array}$ & & 1.00 & $\begin{array}{l}0.24 \\
* *\end{array}$ & $\begin{array}{l}-0.59 \\
* * *\end{array}$ & $\begin{array}{l}0.41 \\
* * *\end{array}$ & $\begin{array}{l}0.50 \\
* * *\end{array}$ & $\begin{array}{l}0.37 \\
* * * *\end{array}$ \\
\hline $\begin{array}{l}\text { Number of } \\
\text { Applications }\left(\mathrm{MT}_{\mathrm{C}}\right)\end{array}$ & & & 1.00 & $\begin{array}{l}0.43 \\
* * * *\end{array}$ & $\begin{array}{l}0.48 \\
* * *\end{array}$ & $\begin{array}{l}0.29 \\
* *\end{array}$ & $\begin{array}{l}0.24 \\
* *\end{array}$ \\
\hline Number of Appl. by Task (MT $\left.\mathrm{MC}_{\mathrm{TC}}\right)$ & & & & 1.00 & 0.05 & $\begin{array}{l}-0.33 \\
* * *\end{array}$ & $\begin{array}{l}-0.27 \\
* *\end{array}$ \\
\hline $\begin{array}{l}\text { Number of } \\
\text { Windows }\left(\mathrm{MT}_{\mathrm{UC}}\right)\end{array}$ & & & & & 1.00 & $\begin{array}{l}0.30 \\
* * *\end{array}$ & 0.14 \\
\hline $\begin{array}{l}\text { Percentage of } \\
\text { Overlapping Tasks }\left(\mathrm{MT}_{\mathrm{UT}}\right)\end{array}$ & & & & & & 1.00 & $\begin{array}{l}0.70 \\
* * *\end{array}$ \\
\hline $\begin{array}{l}\text { Percentage of } \text { Between-Task } \\
\left.\text { Switches (MT } \mathrm{MTC}_{\mathrm{UTC}}\right)\end{array}$ & & & & & & & 1.00 \\
\hline
\end{tabular}

Significance Levels: $* \mathrm{p}<.05 ; * * \mathrm{p}<.01 ; * * * \mathrm{p}<.001$.

Table III. Percentage of Between-Task Switching by Multitasking Category

\begin{tabular}{|l|c|c|c|}
\hline $\mathrm{n}=146$ & $\begin{array}{c}\text { Non-Multitaskers } \\
(\mathrm{n}=70)\end{array}$ & $\begin{array}{c}\text { Multitaskers } \\
(\mathrm{n}=76)\end{array}$ & $\begin{array}{c}\text { Kruskal-Wallis } \\
\text { Test (Chi-Square) }\end{array}$ \\
\hline $\begin{array}{l}\text { Percentage of overlapping tasks } \\
\text { MT }_{\text {UT }}\end{array}$ & $39(30)$ & $70(17)$ & $41.15^{* * *}$ \\
\hline $\begin{array}{l}\text { Percentage of between-task switches } \\
\text { MT }_{\text {UTC }}\end{array}$ & $17(16)$ & $42(17)$ & $56.44^{* * *}$ \\
\hline
\end{tabular}

Cells show means and standard deviation in parentheses. Significance: $* * * p<.001$.

ranges from 0 to $81 \%$ with a mean of $30 \%$ and a standard deviation of $21 \%$. This measure is indicative of the overall strategy employed by participants in combining multiple tasks in their computer-usage sessions. Users who are purely sequential do not have any between-task switches, while those who interleave independent tasks have a high proportion of between task switches.

We compared the metrics determined by the log-analysis program with a correlation analysis that included session length. The results, shown in Table II, indicate that session duration is not significantly correlated with any of our multitasking metrics. According to these findings, neither number of tasks nor multitasking patterns are influenced by the length of the session.

The richest multitasking metric $\left(\mathbf{M} \mathbf{T}_{\mathrm{UTC}}\right)$ is significantly correlated with number of tasks $\mathbf{M} \mathbf{T}_{\mathrm{T}}(\rho=0.37, \mathbf{p}<.001)$, with number of applications $\mathbf{M} \mathbf{T}_{\mathrm{C}}(\rho=0.24, \mathbf{p}<.01)$, and with percentage of overlapping tasks $\mathbf{M} \mathbf{T}_{\mathrm{UT}}(\rho=0.70, \mathbf{p}<.001)$, indicating that this metric contains elements of task, user and technology. Additionally, the high and significant correlation $(\rho=0.70, \mathrm{p}<.001)$ between the percentage of overlapping tasks $\left(\mathbf{M T}_{\mathrm{UT}}\right)$ and the switches-based measure $\left(\mathbf{M T}_{\mathrm{UTC}}\right)$ suggests that both give an indication of concurrency, the former from the perspective of the tasks, and the latter from the perspective of the users' switching behavior.

In order to validate these two metrics (MT $\mathbf{M T}_{\mathrm{UT}}$ and $\mathbf{M T}_{\mathrm{UTC}}$ ), we compared them with the multitasking indicator determined by human coders $\left(\mathbf{M} \mathbf{T}_{U}\right)$ using a Kruskal-Wallis test (See Table III). We find a significant association between dichotomous multitasking $\left(\mathbf{M} \mathbf{T}_{\mathrm{U}}\right)$ determined by the coders and each of these measures, which were computed by the log-analysis program. Multitaskers have a significantly higher percentage of tasks overlapping than non-multitaskers $(70 \%$ vs. $39 \%$; Chi-Square $=41.15 ; \mathrm{p}<.001)$. In addition, those who are multitasking according to the coders' assessment also have a significantly higher percentage of between-task switches than those who are not (17\% vs. $42 \%$; Chi-Square $=56.44 ; \mathrm{p}<.001$ ). Therefore, these richer multitasking metrics indicate the extent to which multitasking is occurring. 
Although a non-multitasking user should have no overlapping tasks and zero between-task switches, the behavior exhibited by the users and captured in the logs is mixed with respect to the temporal organization of tasks. Put differently, nonmultitaskers had a minimal amount of task overlap, low percentages of between task switches, and their predominant task performance behavior was sequential.

\section{DISCUSSION}

The metrics we propose were tested with data from a sample of users who selfreported their computer-based activities. User-produced logs were analyzed by human coders who determined the lean metric (dichotomous multitasking), and by a customdeveloped log-analysis program in Perl that computed the other measures. From the set of richer metrics that incorporate at least two of the elements of the triad, we find that number of windows ( $\left.\mathbf{M T} \mathbf{T C}_{\mathrm{UC}}\right)$ and number of applications by task $\left(\mathbf{M T} \mathbf{T}_{\mathrm{TC}}\right)$ are limited in their ability to indicate concurrent execution of unrelated tasks. In contrast, the overlap measure $\left(\mathbf{M T}_{\mathrm{UT}}\right)$ gives an indication of performance concurrency; and the switches-based metric (MT $\mathbf{M T C}_{\mathrm{UTC}}$ ) indicates the extent to which user attention shifts among unrelated ongoing activities. These two metrics are not only more informative but also consistent with our broad conceptualization of multitasking in terms of task independence and performance concurrency.

Additionally, we found that these two metrics ( $\mathbf{M} \mathbf{T}_{\mathrm{UT}}$ and $\mathbf{M} \mathbf{T}_{\mathrm{UTC}}$ ), determined by our automatic coding program, are significantly correlated with the leanest measure $\left(\mathbf{M T}_{\mathrm{U}}\right)$ determined by human coders. This significant association provides evidence of the validity of $\mathbf{M} \mathbf{T}_{\mathrm{UT}}$ and $\mathbf{M} \mathbf{T}_{\mathrm{UTC}}$ given our definition of computer-based multitasking behavior. In fact, the empirical evaluation and comparison of metrics shows that the users who were multitasking have a high percentage of overlapping tasks (70\%) and an average proportion of between-task switches of $42 \%$. In contrast, those who were not multitasking have a significantly lower percentage of overlapping tasks (39\%) and a significantly lower percentage of between-task switches (17\%).

In theory, when a user is not multitasking (his/her $\mathbf{M T}_{\mathrm{U}}$ is zero), s/he should not have any overlapping tasks, nor should s/he exhibit any between task switches. In this case, both $\mathbf{M} \mathbf{T}_{\mathrm{UT}}$ and $\mathbf{M} \mathbf{T}_{\mathrm{UTC}}$ should be zero. However, we noticed two patterns of user behavior that produced some task overlaps and consequently higher than zero counts of between-task switches in non-multitaskers. First, some users occasionally switched to an unrelated task but their predominant pattern during the session was to stay on the same task. Although there was some evidence of minor switching among unrelated tasks, most of the switches were within the same task. Proportionally speaking, Sb was minimal when compared to Sw. Second, users occasionally finished a task without closing the windows associated with it. The tendency to leave windows open even when they were no longer needed and to close all the windows at the end of the session added some additional between task switches, even when the final switch was just to close the window. These two patterns of user behavior (mixed temporal strategies and window neglect) explain the $39 \%$ task overlap findings and the $17 \%$ average proportion of between-task switches in subjects who were not multitasking shown in Table III.

Although both measures (percentage of overlapping tasks ( $\mathbf{M T}_{\mathrm{UT}}$ ) and proportion of between-task switches $\left(\mathbf{M T}_{\mathrm{UTC}}\right)$ ) indicate concurrency, the latter is more accurate in measuring the extent to which the users shift their focus among ongoing but unrelated tasks. In other words, the proportion of between-task switches is the best measure to capture and reflect the strategy employed by the user to combine multiple computerbased tasks. For example, if a pure sequential approach is followed and a task is initiated only upon the completion of the previous one, our $\mathbf{M} \mathbf{T}_{\mathrm{UTC}}$ multitasking measure is close to zero. In contrast, if the user interleaves independent tasks by switching 
among them frequently, multitasking measured with $\mathbf{M} \mathbf{T}_{\mathrm{UTC}}$ is higher and indicates the extent to which interleaving takes place.

It should be noted that $\mathbf{M} \mathbf{T}_{\mathrm{UTC}}$ is based on an analysis of switching behavior regardless of the triggers of switches. A user may shift the focus of attention from one window to another due to an internal decision or due to an external notification. For instance, a change to a checkbook balancing spreadsheet while doing online banking is an example of a deliberate focus shift because it is an internally triggered self-initiated switch. In contrast, a change of windows to check email while writing a research report could be internally motivated or prompted by an email notification. The latter is an example of a breakdown-induced switch. In this study, we are only interested in the type of switch ( $\mathrm{Sb}$ or $\mathrm{Sw}$ ) with respect to the underlying task, and not on the determinants of switches. We recognize, however, that our switching-related metric can be extended in this direction. The use of focus shift analysis as proposed by Bødker [1996] could be useful to expand this metric.

\subsection{Limitations}

An important observation associated with this analysis is that these metrics are dependent on the quality of the underlying data sources. Our data is based on information reported by the subjects themselves using a standardized paper form. Although the validity of self-reports has been called into question, researchers have found that selfreported estimates are relatively accurate and similar in magnitude to log data values [Deane et al. 1998]. More importantly, self-reported logs have the advantage of allowing users to describe their tasks in their own words. Such an understanding is not available with automatic logs produced by monitoring software. We believe that future research applying these metrics will benefit from a combination of user self-reported logs matched with automatically collected monitoring logs of computer usage.

An additional limitation of our self-report requirements is that the process of manually logging computer-based activity may have affected the flow of work of some participants, and may have caused under-reporting of a number of switches, particularly in rapid switching situations such as those created by synchronous conversations (chats). Consequently, the calculation of the number of switches may not be entirely accurate. Despite possible reporting inaccuracies, it is encouraging that our measures are robust and discriminate between multitaskers and non-multitaskers.

Due to the type of subjects who participated in this study (college students) and the homebound nature of their computer session, we refrain from making any generalizations about multitasking behavior. It is possible that the application of our metrics to other subjects and contexts (for example, professionals in a work environment) would result in different patterns of multitasking behavior. The objective of this empirical study is to demonstrate how to collect and validate the different multitasking measures we are proposing.

Another source of limitations stems from our focus on a single computer platform, which determined that our technology-related metrics $\left(\mathbf{M} \mathbf{T}_{\mathrm{C}}\right.$ and $\mathbf{M} \mathbf{T}_{\mathrm{TC}}$ ) were based on applications. ${ }^{6}$ In $\mathbf{M T}_{\mathrm{C}}$ the technology component was measured with the number applications, which were defined in terms of specific software programs. When tasks are considered, our metric $\mathbf{M} \mathbf{T}_{\mathrm{TC}}$ (number of applications by task) is a ratio that does not give an exact count of the applications for each task. However, as an average, this metric shows the balance of tasks to applications. An extension of this metric could incorporate the distinction between having multiple applications to perform a single

${ }^{6}$ In MTuc the technology component was measured with the number of windows because the decision of how many windows to use is at the discretion of the user. 
task vis-à-vis having multiple applications to switch between several independent tasks. $^{7}$

Although at first glance our metrics appear to be only relevant for a personal computer environment, our focus on temporal integration for determining task overlap for $\mathbf{M T}_{\mathrm{UT}}$ and on switches for $\mathbf{M} \mathbf{T}_{\mathrm{UTC}}$ allows for an easy extension of these metrics to other technology environments or to situations where multiple devices are used. On the one hand, due to its focus on the user-task pair the overlap measure ( $\left.\mathbf{M T}_{\mathrm{UT}}\right)$ can be useful for the analysis of computer-based and non-computer based tasks. On the other hand, the switches-based measure can be applied to environments where multiple technologies are used, including mobile devices. In such "media multitasking" [Ophir et al. 2009] environments, between-task and within-task switching measures can be extended to account for whether the underlying technology device used has changed.

\subsection{Implications for Theory, Research, and Practice}

The set of metrics proposed and evaluated in this study are aimed at quantifying multitasking from the perspective of Activity Theory and the user-task-technology triad. These concepts provide the foundation to develop richer multitasking metrics. The richest measure we propose $\left(\mathbf{M T}_{\mathrm{UTC}}\right)$ takes into account the perspective of the task, the user and the technology simultaneously. It is based on the articulation of switches in two categories, namely between-task switches $(\mathrm{Sb})$ and within-task switches ( $\mathrm{Sw}$ ). The notion of between-task switches is paramount, as it provides an indication of the user's decisions to change tasks. $\mathbf{M T}_{\mathrm{UTC}}$ captures the extent to which the user switches, regardless of the triggers of such switches. Whether a change of task is motivated by a notification, or an interruption or an internal decision to change tasks, this metric only considers the switches and the relatedness of the abandoned task with respect to the new task. The focus on switches as evidence of time allocation decisions in a multitasking context is a key consideration to integrate the existing body of literature on interruptions and discretionary task interleaving. By placing the focus on the decision to change tasks (actual switches), this unifying metric can be helpful to integrate these two streams of the literature.

In addition, $\mathbf{M T} \mathbf{T}_{\mathrm{UTC}}$ allows for an analysis of the interruptions literature from a new perspective. Empirical findings on interruptions indicate that switching between related tasks is more beneficial than switching between unrelated tasks [Cutrell et al. 2000]. Furthermore, a high frequency of unrelated interruptions has a detrimental effect on performance [Speier et al. 2003]. In the context of our proposed definitions and metric, related switching (Sw) does not affect multitasking measured with $\mathbf{M T}_{\mathrm{UTC}}$, while Sb switching does increase the amount of multitasking. This insight provided by our $\mathbf{M T}_{\mathrm{UTC}}$ metric used in conjunction with focus shift analysis [Bødker 1996] offers a fruitful roadmap to investigate multitasking in more depth.

For HCI designers, knowing whether or not people multitask is helpful, but identifying the extent to which multitasking occurs can be even more valuable. Besides using our metrics to determine how much multitasking takes place in a given session, these metrics can also be used to examine multitasking within particular applications, and determine when users employ specific applications in conjunction with others. Interaction designers can use this knowledge to improve the design of applications that are typically used simultaneously, or the design of interfaces that support multitasking. It is also possible to use our metrics to develop programs that specifically measure and monitor multitasking behavior. Such programs could be particularly useful in work environments.

\footnotetext{
${ }^{7}$ We are grateful to an anonymous conference reviewer for pointing out this issue.
} 
For practitioners our metrics are also relevant, not only to examine their own multitasking patterns, but also to supervise the work of others. An understanding of how multitasking occurs is the first step to appreciate the limits of multitasking and its effect on performance. It is possible that high percentages of multitasking are conducive to poor performance, which may have severe consequences in actual work environments. More research is needed to investigate the relation between multitasking and performance with the metrics proposed in this study.

In light of these implications, future research can expand this study in several theoretical, methodological and empirical directions. First, there is ample opportunity to solidify the theoretical roots of multitasking behavior using Activity Theory or other theories. This work will further enhance our understanding of this prevalent behavior. Second, to improve the methodological foundations in support of multitasking research, additional objective metrics can be developed, tested and compared to the ones presented here. A parallel effort could consist of using our definition of multitasking and the triad to develop subjective measures or scales to assess the perception of multitasking, or typical multitasking habits. For example, Ophir et al. [2009] recently developed a media multitasking index to capture the concurrent use of different media. A similar index could be developed to measure the propensity to undertake several independent tasks concurrently. Finally, our metrics can be applied in a larger sample of users by collecting self-reported logs along with system-generated logs to investigate multitasking behavior patterns. In addition, qualitative studies of user-generated logs with focus shift analysis as used in Bødker [1996] can offer new insights into the reasons behind multitasking.

The main contribution of this work is twofold. First, it provides an encompassing definition of multitasking in terms of task independence and performance concurrency. Consensus on the definition of multitasking enables researchers to integrate the existing literature on this topic and paves the way to develop a nomological network for multitasking. Second, the structure and operationalization of computer-based multitasking offers concrete metrics to enhance current studies of IT usage and a framework to develop other measures. Future multitasking studies would benefit from such a strong methodological foundation.

\section{CONCLUSION}

This study lays the groundwork to investigate multitasking and integrate the literature on interruptions and discretionary task switching. With the theoretical background of Activity Theory and the three elements proposed to study IT usage (user, technology and tasks), we developed different multitasking metrics for computer-based multitasking behavior. We then conducted a study to assess the validity of the proposed metrics and concluded that a very simple dichotomous variable offers an indication of whether or not the user is multitasking, while a richer metric based on an analysis of switches indicates the extent to which a user is multitasking. The main contribution of this paper is to conceptualize multitasking and to offer alternative measures to investigate this behavior in a computer-based environment. This new conceptualization and metrics open up multiple avenues of inquiry aimed at achieving a better understanding of multitasking behavior.

\section{APPENDIX A. Instructions for Participants}

Choose a session when you will be using your computer at home for a purpose that does not include doing this assignment. You can be doing an assignment for another class or using your computer for leisure. Try to select a session that is representative of your typical computer usage and whose duration is approximately one or two hours. 
Keep a detailed log of your computer-based activities. Using the printed form distributed in class, fill out the time (e.g. 7:34pm), whether you open, close or return to a previously open window, the applications you open (e.g. Word, Excel, Browser, or browser tabs) and the files you open in any of these applications (if applicable). Also, write down what you do in each window and the reasons for switching to another window. Please fill out the log manually as you are using your computer, so it does not interfere with your normal computer-based activities. Your log should show a detailed account of your activities.

\section{APPENDIX B. Log Example}

\begin{tabular}{|c|c|c|c|c|c|}
\hline $\begin{array}{l}\text { Time } \\
\text { Stamp }\end{array}$ & Action & Application & $\begin{array}{l}\text { File or Tab } \\
\text { Or WindowTitle }\end{array}$ & $\begin{array}{l}\text { Purpose } \\
\text { on window }\end{array}$ & $\begin{array}{l}\text { Reason for going } \\
\text { to another window }\end{array}$ \\
\hline $17: 30$ & Open & $\begin{array}{l}\text { Mozilla } \\
\text { Browser }\end{array}$ & Yahoo.com & $\begin{array}{l}\text { Home page } \\
\text { (news/search engine }\end{array}$ & $\begin{array}{l}\text { Need to check my email on } \\
\text { different Website }\end{array}$ \\
\hline $17: 35$ & Open & Browser & Tab (Gmail.com) & Check email & Read my email \\
\hline 17:41 & Open & Browser & Tab (Nba.com) & Check NBA scores & Need to check email \\
\hline $17: 45$ & Open & Browser & Tab (hotmail.com) & Check email & Back to looking at NBA \\
\hline $17: 50$ & Close & Browser & Tab (Nba.com) & NBA scores & Done looking at it \\
\hline $17: 53$ & Switch & Browser & Tab (Hotmail.com) & Email & Go to social network site \\
\hline $17: 56$ & Open & Browser & Facebook & Social Networking & $\begin{array}{l}\text { Open up instant messen- } \\
\text { ger }\end{array}$ \\
\hline 18:02 & Open & Aim & IM & Instant messaging & Edit Facebook \\
\hline $18: 15$ & Switch & Browser & Facebook & Editing & Instant Message received \\
\hline $18: 16$ & Switch & Aim & IM & Instant messaging & Received Notification \\
\hline $18: 21$ & Switch & Browser & Facebook & Social Networking & Instant message \\
\hline $18: 22$ & Switch & Aim & $\mathrm{IM}$ & Instant messaging & Link received from Friend \\
\hline $18: 28$ & Open & Browser & Youtube & Video sharing & Instant message \\
\hline $18: 29$ & Switch & Aim & IM & Instant messaging & Check out video \\
\hline 18:33 & Switch & Browser & Youtube & Video sharing & Instant message \\
\hline 18:34 & Switch & Aim & IM & Instant messaging & Received notification \\
\hline $18: 40$ & Switch & Browser & Facebook & Edit site & Instant message \\
\hline 18:41 & Switch & Aim & IM & Instant messaging & Open music player \\
\hline $18: 50$ & Open & $\begin{array}{l}\text { Windows } \\
\text { Media player }\end{array}$ & File < user>'s playlist & Music player & Instant message \\
\hline 18:51 & Switch & Aim & IM & Instant messaging & Close Facebook \\
\hline $18: 53$ & Close & Browser & Facebook & Social Networking & Instant message \\
\hline $18: 54$ & Switch & Aim & $\mathrm{IM}$ & Instant messaging & Close Gmail \\
\hline $18: 57$ & Close & Browser & Gmail.com & Email & Instant message \\
\hline $18: 58$ & Switch & Aim & IM & Instant messaging & Change music \\
\hline 19:00 & Switch & WMP & $<$ user>'s playlist & Music player & Instant Message \\
\hline
\end{tabular}

\section{APPENDIX C. List of Tasks}

\begin{tabular}{|l|c|}
\hline Task & Percentage of logs \\
\hline Academic (doing homework, using courseware system, preparing for class, etc.) & $82 \%$ \\
\hline Email & $80 \%$ \\
\hline Chat, Instant messaging (IM) & $52 \%$ \\
\hline Social Networking & $48 \%$ \\
\hline Running music player program & $40 \%$ \\
\hline Reading Web-based news & $32 \%$ \\
\hline Watching movies, TV programs or video clips on the computer & $29 \%$ \\
\hline Online shopping & $22 \%$ \\
\hline Online banking & $18 \%$ \\
\hline Checking Sports scores, teams, stats & $10 \%$ \\
\hline Playing computer games & $8 \%$ \\
\hline Scheduling (activities, to-do list, appointments, calendar, etc.) & $8 \%$ \\
\hline Checking weather & $8 \%$ \\
\hline Photos (editing, uploading, downloading, organizing) & $5 \%$ \\
\hline
\end{tabular}




\section{ACKNOWLEDGMENTS}

This study originated from the class discussions held in the Doctoral Seminar "Research in Information Systems" taught by R. Benbunan-Fich at Baruch College in the Spring of 2008. The first version of this study was presented as a research-in-progress poster at SIG-CHI 2009, and an extended abstract was published in their proceedings. Subsequently, a full paper entitled "Developing New Metrics for ComputerBased Multitasking Behavior" was accepted and presented at ICIS 2009-Breakthrough Ideas Track. The current manuscript has benefited from the feedback received at these conferences. In addition, the authors would like to thank the Associate Editor and the anonymous reviewers of this journal for their helpful comments and suggestions to refine the presentation of this innovative work.

\section{REFERENCES}

Bailey, B. P. AND IqBal, S. T. 2008. Understanding changes in mental workload during execution of goaldirected tasks and its application for interruption management. ACM Trans. Comput.-Hum. Interact. $14,4,21-56$.

Bell, C. S., Compeau, D. R., and Olivera, F. 2005. Understanding the social implications of technological multitasking: A conceptual model. In Proceedings of the 4th Annual Worskshop on HCI Research in MIS. 80-84.

Bedny, G. Z., Chebykin, O., ANd Karwowski, W. 2005. The task as a basic object of study in an HCI system. In Proceedings of the 11th International Conference on Human-Computer Interaction (HCI'05). M. J. Smith and G. Salvendy Eds., Lawrence Erlbaum Associates, Mahwah, NJ.

BeDny, G. Z. AND HaRRis, S. R. 2005. The systemic-structural theory of activity: Applications to the study of human work. Mind, Cult. Activ. 12, 2, 128-147.

BEDNY, G. Z. AND HARRIS, S. R. 2008. "Working sphere/engagement" and the concept of task in activity theory. Interact. Comput. 20, 251-255.

Bedny, G. Z. And Karwowski, W. 2007. A Systemic-Structural Theory of Activity: Applications to Human Performance and Work Design. CRC/Taylor \& Francis, Boca Raton, FL.

BEDNY, G. Z. AND Karwowski, W. 2003. A systemic-structural activity approach to the design of humancomputer interaction tasks. Int. J. Hum.-Comput. Interact. 16, 2, 235-260.

Bedny, G. Z., Seglin, M. H., And Meister, D. 2000. Activity theory: History, research and application. Theor. Iss. Ergon. Sci. 1, 2, 168-206.

Benbunan-Fich, R., AND Truman, G. E. 2009. Multitasking with laptops during meetings. Comm. ACM, 52, 2, 139-141.

Bluedorn, A. C., Kaufman, C. F., and Lane, P. M. 1992. How many things do you like to do at once? An introduction to monochronic and polychronic time. Acad. Manage. Exec. 6, 4, 17-26. Bødker, S. 1989 . A human activity approach to user interfaces. Hum.-Comput. Interact. 4, 3, 171-195.

BøDKER, S. 1996. Applying Activity Theory to Video Analysis: How to make sense of video data in HumanComputer Interaction. In Context and Consciousness, Nardi, B. A. Ed., MIT Press, Cambridge, MA, 147-174.

Burton-Jones, A. and Straub, D. W. 2006. Reconceptualizing system usage: An approach and empirical test. Inform. Syst. Resear. 17, 3, 228-246.

Crook, C. AND BarrowClifF, D. 2001. Ubiquitous computing on campus: Patterns of engagement by university students. Int. J. Hum.-Comput. Interact. 13, 2, 245-256.

Cutrell, E. B., Czerwinski, M., ANd Horvitz, E. 2000. Effects of instant messaging interruptions on computing tasks. In Proceedings of the SIGCHI Conference on Human Factors in Computing Systems. 99-100.

Czerwinski, M., Horvitz, E., AND Wilhite, S. 2004. A diary study of task switching and interruptions. In Proceedings of the SIGCHI Conference on Human Factors in Computing Systems. 175-182.

Deane, F. P., Podd, J., And Henderson, R. D. 1998. Relationship between self-report and log data estimates of information system usage. Comput. Hum. Behav. 14, 4, 621-636.

Gillie, T. And Broadbent, D. 1989. What makes interruptions disruptive? A study of length, similarity, and complexity. Psych. Resear. 50, 4, 243-250.

GonZÁLEZ, V. M. AND MARK, G. 2004. "Constant, constant, multi-tasking craziness": Managing multiple working spheres. In Proceedings of the SIGCHI Conference on Human Factors in Computing Systems. $113-120$.

HACKman, J. R. 1969. Toward understanding the role of tasks in behavioral research. Acta Psychologica, 31, 97-128.

Hall, E. T. 1983. The Dance of Life: The Other Dimension of Time. Anchor Books/Doubleday, Garden City, NY. 
Hembrooke, H. AND GAY, G. 2003. The laptop and the lecture: The effects of multitasking in learning environments. J. Comput. Higher Ed. 15, 1, 46-64.

HodgetTs, H. M. AND Jones, D. M. 2006. Interruption of the Tower of London task: Support for a goal activation approach. J. Exper. Psych. Gen. 135, 1, 103-115.

KAPTELININ, V. 1996. Activity theory: Implications for human-computer interaction. In Context and Consciousness, Nardi, B. A. Ed., MIT Press, Cambridge, MA, 53-59.

Kaptelinin, V. And Nardi, B. A. 2006. Acting with Technology: Activity Theory and Interaction Design. MIT Press, Cambridge, MA.

KuUTTI, K. 1991. Activity theory and its applications to information systems research and development. In Information Systems Research, H.-E. Nissen Ed., Elsevier Science Publishers, Amsterdam, 529-549.

KuUTTI, K. 1996. Activity Theory as a potential framework for Human-Computer Interaction. In Context and Consciousness, Nardi, B. A. Ed., MIT Press, Cambridge, MA, 17-44.

LeE, H. 1999. Time and information technology: Monochronicity, polychronicity and temporal symmetry. Eur. J. Inform. Syst. 8, 1, 16-26.

Mark, G., GonzÁlez, V. M., AND HARRIS, J. 2005. No task left behind?: Examining the nature of fragmented work. In Proceedings of the SIGCHI Conference on Human Factors in Computing Systems. 321-330.

McCrickard, D. S., Chewar, C. M., Somervell, J. P., and Ndiwalana, A. 2003. A model for notification systems evaluation-Assessing user goals for multitasking activity. ACM Trans. Comput.-Hum. Interact.,10, 4, $312-338$.

McFarLANE, D. C. 2002. Comparison of four primary methods for coordinating the interruption of people in human-computer interaction. Hum.-Comput. Interact. 17, 1, 63-139.

McFarlane, D. C. and Latorella, K.A. 2002. The scope and importance of human interruption in humancomputer interaction design. Hum.-Comput. Interact. 17, 1, 1-61.

McGrath, J. E. 1991. Time, interaction and performance (TIP): A theory of groups. Small Group Resear. 22, $2,147-174$

NARDI, B. A. 1996. Studying context: A comparison of activity theory, situated action models and distributed cognition. In Context and Consciousness, Nardi, B. A. Ed., MIT Press, Cambridge, MA, 69-102.

Ophir, E., Nass, C., AND Wagner, A. D. 2009. Cognitive control in media multitaskers. Proc. Nat. Acad. Sci. $1-5$.

Payne, S. J., Duggan, G. B., and Neth, H. 2007. Discretionary task interleaving: Heuristics for time allocation in cognitive foraging. J. Exper. Psych. Gen. 136, 3, 370-388.

Reinsch, N.L., Turner, J. W., And Tinsley, C. H. 2008. Multicommunicating: A practice whose time has come?" Acad. Manage. Rev. 33, 2, 391-403.

Renaud, K., Ramsay, J., ANd HaIR. M. 2006. "You've got e-mail!”... Shall I deal with it now? Electronic mail from the recipient's perspective. Int. J. Hum.-Comput. Interact.,21, 3, 313-332.

Rubinstein, J. S., Meyer, D. E., And Evans, J. E. 2001. Executive control of cognitive processes in task switching. J. Exper. Psych. Hum. Percept. Perform. 27, 4, 763-797.

SalvucCI, D. D. 2009. Rapid prototyping and evaluation of in-vehicle interfaces. ACM Trans. Comput.-Hum. Interact. 16, 2, 1-33.

Speier, C., Vessey, I., And Valacich, J. S. 2003. The effects of interruptions, task complexity, and information presentation on computer-supported decision-making performance. Decis. Sci. 34, 4, 771-797.

Speier, C., Vessey, I., and Valacich, J. S. 1999. The influence of task interruption on individual decision making: An information overload perspective. Decis. Sci. 30, 2, 337-361.

Wasson, C. 2004. Multitasking during virtual meetings. Hum. Resource Plan. 27, 4, 47-61.

WhitTAKer, S. 2005. Supporting collaborative task management in e-mail. Human-Comput. Interact. 20, 1-2, 49-88.

Wild, P. J., Johnson, P., AND Johnson, H. 2004. Towards a composite modeling approach for multitasking. In Proceedings of the 3rd Annual Conference on Task Models and Diagrams. 17-24.

Wood, R. E. 1986. Task complexity: Definition of the construct. Organiz. Behav. Hum. Decis. Proc. 37, 1, 60-82.

Received June 2010; revised September 2010, January 2011; accepted January 2011 